\title{
Erratum to: Hyperaccumulation of trace elements: from uptake and tolerance mechanisms to litter decomposition; selenium as an example
}

\author{
Juan Barceló • Charlotte Poschenrieder
}

Published online: 16 November 2010

(C) Springer Science+Business Media B.V. 2010

\section{Erratum to: Plant Soil \\ DOI 10.1007/s11104-010-0469-0}

The originally published text of this article unfortunately contained a mistake in page 3 , line 3 , right column.

Incorrect: carcinogenic selenium compounds

Correct: anticarcinogenic selenium compounds

Responsible Editor: Hans Lambers.

The online version of the original article can be found at http://dx.doi.org/10.1007/s11104-010-0469-0.

J. Barceló $(\bowtie) \cdot$ C. Poschenrieder

Lab. Fisiología Vegetal, Facultad de Biociencias,

Universidad Autónoma de Barcelona,

08193 Bellaterra, Spain

e-mail: juan.barcelo@uab.es 